\section{Mycobacterium bovis Infection in Animals and Humans, 2nd Edition}

\author{
Charles O. Thoen, James H. Steele, \\ and Michael J. Gilsdorf, editors
}

\section{Blackwell Publishers, Boston, Massachusetts, 2006 \\ ISBN: 0813809193}

Pages: 329; Price: US \$124.99

The stated purpose of the second edition of Mycobacterium bovis Infection in Animals and Humans is to provide medical professionals, allied health scientists, research workers, and graduate students with current information on the significance of $M$. bovis in the control and eradication of tuberculosis in animals and humans. This newest edition deals with topics such as the public health significance of $M$. bovis, pathogenesis of $M$. bovis, epidemiology of $M$. bovis (with an entire chapter on molecular epidemiologic techniques), PCR detection of $M$. bovis with formalinfixed tissues, and DNA vaccines. As with the first edition, the second edition delivers several updates from various countries on the status of $M$. bovis infection in animals and humans. Little accessible published information has been available on this topic, which makes the book especially useful.

The second edition also deals with several areas not covered in the first edition, including molecular epidemiology, evolution of the M. tuberculosis complex, tuberculosis caused by $M$. pinnipedii in fur seals and sea lions, the economics of bovine tuberculosis, and cost-benefit analysis of disease eradication programs. Several chapters deal with timely issues related to tuberculosis in wildlife.
In spite of its strengths, the second edition adds little additional information to material provided in the first edition on the topics of pathogenesis or diagnosis of bovine tuberculosis. In addition, although most of the photomicrographs are adequate, several are of such poor quality that they are of little use. Also, as with any multiauthored volume, some repetition occurs on general topics. The book achieves its stated purposes, however, and will be especially useful as a reference for researchers, regulatory agencies, and graduate students. It will be less informative for those interested in detailed discussions on research in the field of pathogenesis or diagnosis of $M$. bovis infection.

\section{Mitchell V. Palmer*}

*US Department of Agriculture, Ames, Iowa, USA

Address for correspondence: Mitchell V. Palmer, National Animal Disease Center, US Department of Agriculture, 2300 Dayton Ave, Ames, IA 50010, USA; email: mpalmer@nadc. ars.usda.gov

\section{Evolution of Microbial Pathogens}

\section{H. Steven Seifert and Victor J. DiRita, editors}

\section{American Society for Microbiology Press, Washington, DC, 2006 ISBN: 1-55581-300-3 \\ Pages: 355; Price: US \$119.95}

This book is one of the first to provide an up-to-date view on a fundamental issue in medical microbiology research: how the accumulated genetic and genomic information is contributing to our understanding of virulence factors and the evolution of virulence in microbial pathogens. The editors should be commended for assembling 35 outstanding contributors, who specialize in various areas of microbial pathogenesis and evolution.

The 14 chapters are grouped into 3 broad sections: general concepts in microbial evolution, environment and the evolution of microbial pathogens, and the evolution of selected pathogenic species and mechanisms. At the beginning of each section, a concise overview of individual chapters integrates the content of the chapters into the section.

In the first section, the 5 chapters introduce the basic processes affecting microbial evolution, from the individual molecular level to the genomic, cellular, and population levels. Well-known concepts such as horizontal (lateral) gene transfer, the relationship between virulence and transmission, and pathogenicity islands are discussed extensively. Of special note are 2 chapters that are often missing in traditional medical microbiology books: 1 describes how long-term experimental evolutionary studies in the laboratory can contribute to our understanding of microbial pathogen evolution in the environment and clinics, and the other describes how gene inactivation and gene loss can be creative forces during the evolution of many microorganisms, especially obligate intracellular pathogens.

In the second section, the 5 chapters review how interactions between microbes and various natural biotic and abiotic factors can influence the origin and evolution of virulence in microbial pathogens. These factors are the physical, chemical, and biologic properties of the soil environment; the plant and animal environments; and to a lesser extent, the 